\title{
Case Report: A Rare Case of Nasal Forehead Mass in Kimura's Disease
}

\author{
He Zhao, Zhi-wei Cao and Zhao-wei Gu* \\ Department of Otolaryngology Head and Neck Surgery, Shengjing Hospital of China Medical University, Shenyang, China
}

Background: Kimura's disease is a rheumatic immune disease and head and neck lymph nodes are often involved. A mass occurring in the nasal forehead is rare. Good prognosis after surgical resection by glucocorticoid therapy is more rare.

Case Summary: We report the rare case of a nasal forehead mass in a 45 -year-old male patient with Kimura's disease. The patient underwent resection of the mass in October 2018 in a local hospital and the postoperative pathology was unclear. He then underwent a second resection in our department in December 2019 mainly because growth of the mass was affecting his appearance. Postoperative pathology confirmed that the patient had Kimura's disease, and he accepted systemic treatment with prednisone. We followed the patient for 10 months after surgery. He is now recovering well and continues to be closely monitored during follow-up.

OPEN ACCESS

Edited by:

Claudio Roberto Cernea, University of São Paulo, Brazil

Reviewed by:

Zahoor Ahmad,

Counties Manukau District Health

Board, New Zealand

Yushi Ueki,

Niigata University, Japan

*Correspondence:

Zhao-wei Gu

zwgu@cmu.edu.cn

Specialty section:

This article was submitted to

Otorhinolaryngology - Head and Neck

Surgery,

a section of the journal

Frontiers in Surgery

Received: 25 February 2021

Accepted: 19 April 2021

Published: 21 May 2021

Citation:

Zhao H, Cao Z-w and Gu Z-w (2021)

Case Report: A Rare Case of Nasal

Forehead Mass in Kimura's Disease.

Front. Surg. 8:672291.

doi: 10.3389/fsurg.2021.672291
Conclusion: It is rare that the painless mass in the nasal forehead is diagnosed as a Kimura's disease.After completely resection of the mass and systemic treatment with prednisone, the patient had a good outcome. We provide experience for the treatment of Kimura's disease in nasal forehead.

Keywords: Kimura disease, surgery, nasal forehead mass, case report, prednisone

\section{INTRODUCTION}

Kimura's disease is a rare, chronic inflammatory disease of unknown etiology that occurs rarely in the nose and often affects the lymph nodes. Its clinical manifestations vary according to the location and size of the masses, which are mainly painless with progressive growth (1). Recently, a patient with Kimura's disease of the forehead above the nose was admitted to our ward. The patient's clinical data are described.

\section{CASE PRESENTATION}

\section{Chief Complaints}

A 45-year-old male patient presented with complaints of a nasal forehead mass which had gradually increased in size over the past 5 years and influenced his appearance.

\section{History of Present Illness}

The patient found the nasal-root mass 5 years ago. The mass was the size of a peanut, soft, of normal skin color and with a swollen smooth surface, often exhibiting self-absorption with no other discomfort. In October 2018, nasal mass resection was performed at a local hospital and postoperative pathology was unclear. A relapse occurred in January 2019 and the mass gradually increased in size over the next 10 months, to approximately the size of $5 \mathrm{~cm} \times 2.5 \mathrm{~cm}$. The patient came to our department for a clear diagnosis and treatment. 


\section{History of Past Illness}

The patient was in good health and had no history of chronic disease. In 2012, he underwent appendectomy in a local hospital due to acute appendicitis.

\section{Physical Examination}

Physical examination of the nose upon admission showed that the shape of the nose was normal. The left part of the nasalroot mass was about the size of $5 \mathrm{~cm} \times 2.5 \mathrm{~cm}$. It was soft, with a smooth surface, no redness or swelling, no ulceration, and no tenderness.

\section{Laboratory Examinations}

No abnormalities in terms of renal function, erythrocyte sedimentation rate (ESR), rheumatism, serum complement (C3/C4), quantitative immunoglobulin determination, immunoglobulin IgG4, antinuclear antibodies or ANCA were found. The possibility of Kimura's disease was not considered before surgery, and IgG4 and IgE levels were not detected before the operation. Positive laboratory results are shown in Table $\mathbf{1 .}$

\section{Imaging Examinations}

After admission, computerized tomography (CT) and magnetic resonance imaging (MRI) examination of the patient's paranasal sinuses were performed. MRI showed an iso-intense signal in the $\mathrm{T} 1$ sequence, iso- and hyper-intense signal in the T2 sequence (Figures 1A-C) and enhancement in the T1 enhanced sequence with an unclear boundary. CT showed a soft tissue signal in the nasal forehead with an unclear boundary (Figure 1D).

\section{Pathological Examinations}

The pathological findings were as follows: in the hyperplastic fibrous tissue, there were hyperplastic lymphoid tissues, lymphoid follicles, a large number of eosinophils between follicles and small hyperplastic blood vessels in the follicles, which focally infiltrated striated muscle (Figure 2). Immunohistochemistry findings were as follows: CD3(+), CD20(+), CD21(FDC+), $\mathrm{CK}(-)$, and Cyclin D1(-).

\section{FINAL DIAGNOSIS}

Kimura's disease of the nasal forehead.

\section{TREATMENT}

The patient underwent surgical decompression of the nasal forehead mass under general anesthesia in December 2019, and then accepted treatment with prednisone following the diagnosis of Kimura's disease. After skin incision, subcutaneous solid tumor could be seen, and the capsule was clear and complete. The tumor was completely removed along the tumor capsule, and the operation cavity was checked, and no residue was found. The surface projection is shown in Figure 3. One month after operation, he was given prednisone $30 \mathrm{mg}$ once a day for 4 weeks and $20 \mathrm{mg}$ once a day for 4 weeks and $10 \mathrm{mg}$ once a day for 4 weeks, total 12 weeks to control the disease, and supplemented with pantoprazole to protect the stomach and a calcium supplement.

\section{OUTCOME AND FOLLOW-UP}

Subcutaneous effusion occurred in the postoperative period and recovered after 1 week. There was no recurrence 6 and 10 months after surgery, and the appearance of the nose and forehead recovered well with acceptable scarring. The relevant images are shown in Figure 3.

\section{DISCUSSION}

Kimura's disease is a rare and benign immune-system disease of unknown etiology that occurs particularly in the maxillofacial region. It is characterized by lympho-proliferative formation of lymphoid follicles and eosinophilic granulomas in soft tissue or lymph nodes (2). Kimura's disease may occur at any age, although the peak age of occurrence is $20-40$ years; the majority of cases occur in middle-aged males $(3,4)$. The patient reported in this article was a 45-year-old male. The first onset of the disease was 5 years ago, after which he underwent resection of the mass in 2018. Kimura's disease should be differentiated from the following diseases: benign tumors, lymphomas, Kaposi sarcoma, and Langerhans cell histiocytosis.

At present, the diagnosis of Kimura's disease depends mainly upon histopathology. CT and MRI are also helpful in the diagnosis of Kimura's disease. Most lesions demonstrate mild or moderate enhancement in post-contrast CT scans and moderate or marked enhancement in post-contrast MR images (5). Tissue infiltration, lymphocyte follicular hyperplasia, fibro-collagenous deposition, and vascular proliferation are the histopathological characteristics of Kimura's disease (6).

In the past, Kimura's disease was sometimes confused with ALHE (angiolymphoid hyperplasia with eosinophilia). ALHE occurs predominantly in the skin, as opposed to Kimura's disease, which occurs as a subcutaneous lesion. Both are characterized

TABLE 1 | Positive laboratory results.

\begin{tabular}{|c|c|c|c|c|}
\hline Test & Before surgery & 1 month after surgery & 4 months after surgery & 10 months after surgery \\
\hline Percentage of eosinophils (Routine blood test) & $27.6 \%$ & $21.4 \%$ & $3.2 \%$ & $1.6 \%$ \\
\hline Eosinophils (Routine blood test) & $2.0 \times 10^{9} / \mathrm{L}$ & $1.7 \times 10^{9} / \mathrm{L}$ & $0.3 \times 10^{9} / \mathrm{L}$ & $0.2 \times 10^{9} / \mathrm{L}$ \\
\hline $\lg G 4 n$ & Not detected & $0.857 \mathrm{~g} / \mathrm{L}$ & $0.553 \mathrm{~g} / \mathrm{L}$ & $0.368 \mathrm{~g} / \mathrm{L}$ \\
\hline $\lg \mathrm{E}$ & Not detected & $>2,500 \mathrm{IU} / \mathrm{mL}$ & $>824 \mathrm{U} / \mathrm{mL}$ & $>764 \mathrm{lU} / \mathrm{mL}$ \\
\hline
\end{tabular}




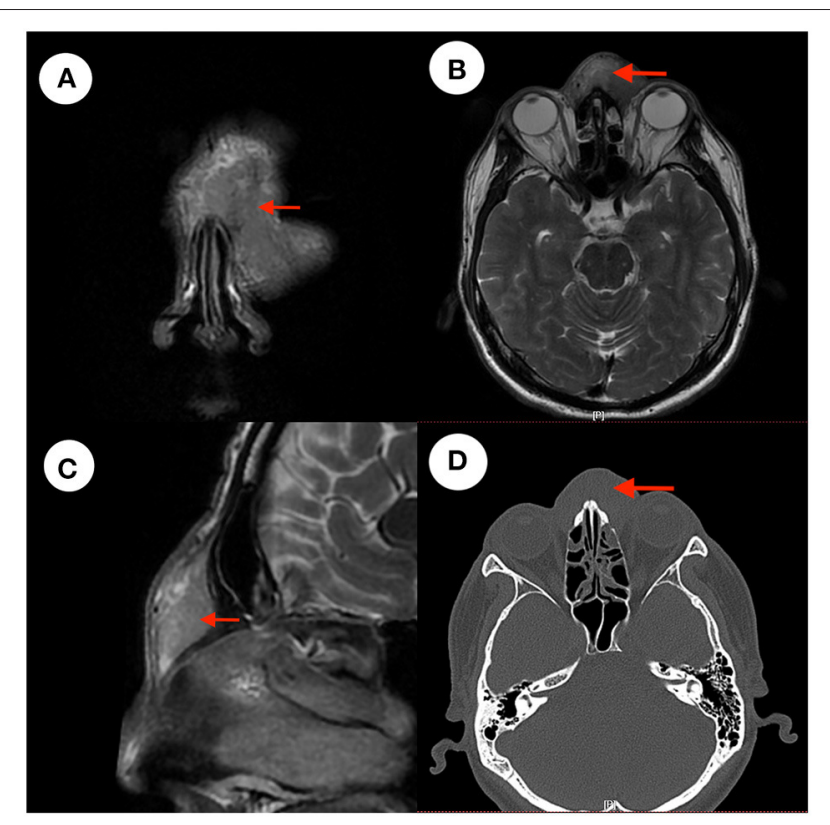

FIGURE 1 | CT and MRI images. (A-C) Show iso- and hyper-intense signal in T2 sequence of the patient's cranium MRI images. Panel $(\mathbf{A})$ is a coronal T2 image. Panel (B) is an axial T2 image. Panel (C) is a sagittal T2 image. Panel (D) is a CT image, with the red arrow indicating the mass.

by heavy eosinophilic infiltration, vascular proliferation and a predilection for appearing in the head and neck region. Conspicuous features of Kimura's disease on histopathology include hyperplastic lymphoid follicles with prominent germinal centers and eosinophilic infiltration. Laboratory tests show that eosinophils on routine blood testing and $\operatorname{IgE}$ in serum are significantly increased in patients with Kimura's disease (6-11). Sometimes, Kimura's disease can occur in other organs, especially the kidneys, where it takes the form of membranoproliferative glomerulonephritis $(12,13)$. The eosinophils on routine blood testing and $\operatorname{IgE}$ in serum are significantly increased, and the diagnosis of Kimura's disease is certified without ALHE.

At present, there is no standard guideline or consensus for the treatment of Kimura's disease. The main treatment for Kimura's disease is surgical removal of the mass and pathological examination, and continue treatment after surgery. According to previous literature, there are hormone therapy, radiotherapy, immunotherapy and treatment of ischemic vascular reconstruction $(14,15)$. For those masses that cannot be completely removed, postoperative radiotherapy is more important. Research has shown that surgical excision combined with low-dose radiotherapy shows better results than surgical excision or radiotherapy alone (16). Because the boundary of the mass was clear and the mass was completely removed during the operation, we did not give the patient radiotherapy after the operation.

Local or systemic steroids can significantly reduce the mass and control the lesions, lymphadenopathy and nephrotic

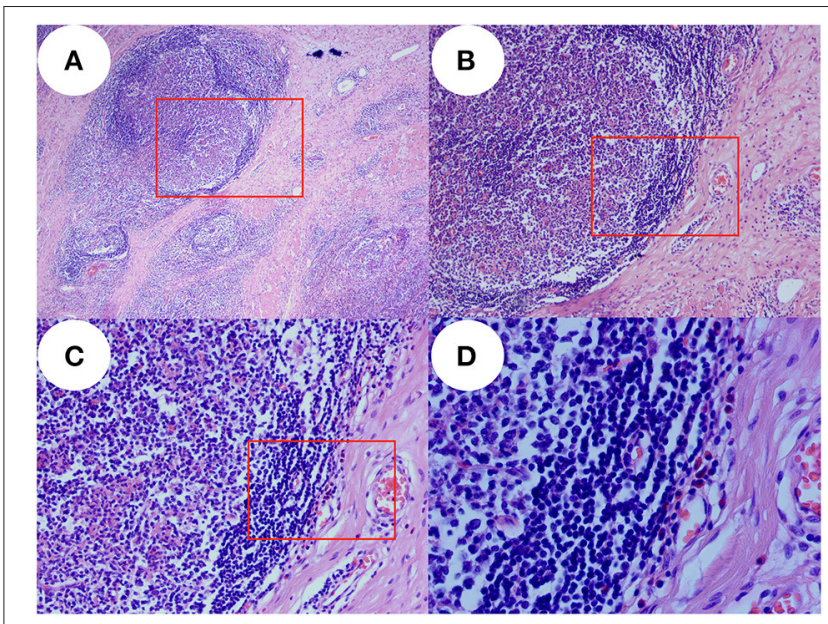

FIGURE 2 | Pathology images. In the proliferative fibrous tissue, there were proliferative lymphoid tissue, lymphoid follicles, a large number of eosinophils between the follicles, proliferative small vessels in the follicles, and local infiltration of striated muscle. Panel $\mathbf{( A )}$ is pathological images taken with an optical microscope at 40x magnification and image (B) was taken at red rectangle. Panel $(\mathbf{B})$ is pathological images taken with an optical microscope at $100 \times$ magnification and image $\mathbf{( C )}$ was taken at red rectangle. Panel $\mathbf{( C )}$ is pathological images taken with an optical microscope at $200 \times$ magnification and image (D) was taken at red rectangle. Panel (D) is pathological images taken with an optical microscope at $400 \times$ magnification which show many eosinophils in the tissue.

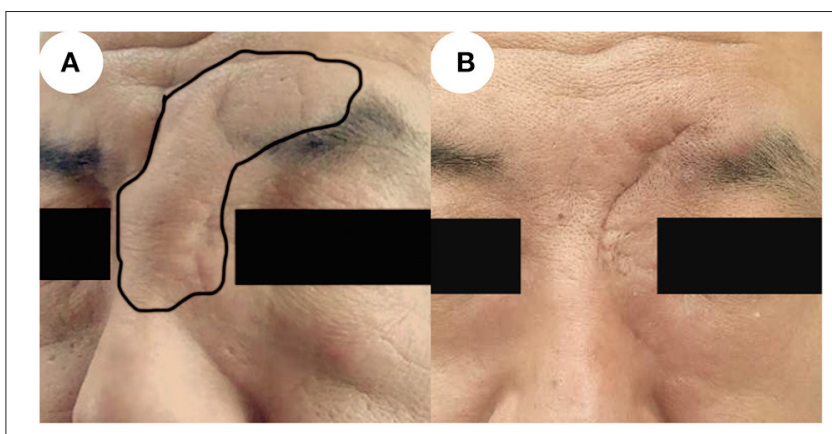

FIGURE 3 | Images before and after surgery. (A) Photo taken prior to surgery, showing the local updrift at the nasal forehead which in the black circle. (B) Six months after surgery, the area is of normal appearance without recurrence.

syndrome (17). Oral prednisolone has a high degree of individualization, and administered in different doses, durations and gradual reduction schemes according to the size of the primary or recurrent lesions (18-20). Even so, the mass also has recurrence after glucocorticoid reduction (about 45.8\%) (21).

Due to the higher eosinophils and IgE in the peripheral blood of the patients, although the mass has been completely removed, after comprehensive consideration, we give the patients oral glucocorticoid therapy, showing a stepwise decline.

Although recurrence is common, no significant recurrence was observed during follow-up in this case. After systemic treatment, eosinophils on routine blood 
testing and $\operatorname{IgE}$ in serum decreased gradually especially the eosinophils which reached normal values. We believe that systemic drug therapy controls eosinophil and IgE levels, and is an important method for preventing postoperative recurrence which the mass was removed completely with high eosinophils and $\operatorname{IgE}$ in the peripheral blood.

\section{CONCLUSION}

Kimura's disease is rare and is mainly diagnosed based on histological examination, as well as physical and radiological examination. Outcome is significantly better after systemic therapy. In the present case, although no recurrence was noted during the 10-month follow-up period, further monitoring is required. Our study demonstrates that a painless nasal forehead mass should be excluded from Kimura's disease and nasal forehead Kimura's disease has a good outcome after surgery and prednisone treatment without radiotherapy.

\section{DATA AVAILABILITY STATEMENT}

The raw data supporting the conclusions of this article will be made available by the authors, without undue reservation.

\section{REFERENCES}

1. Dokania V, Patil D, Agarwal K, Thakur P, Prajapati P. Kimura's disease without peripheral eosinophilia: an unusual and challenging case simulating venous malformation on imaging studies-case report and review of literature. J Clin Diagn Res. (2017) 11:ME01-4. doi: 10.7860/JCDR/2017/28603.10063

2. Wang DY, Mao JH, Zhang Y, Gu WZ, Zhao SA, Chen YF, et al. Kimura disease: a case report and review of the Chinese literature. Nephron Clin Pract. (2009) 111:c55-61. doi: 10.1159/0001 78980

3. Chen C, Chen K, Huang X, Wang K, Qian S. Concurrent eosinophilia and IgG4-related disease in a child: a case report and review of the literature. Exp Ther Med. (2018) 15:2739-48. doi: 10.3892/etm.2018.5743

4. Iguchi $\mathrm{Y}$, Inoue $\mathrm{T}$, Shimono $\mathrm{M}$, Yamamura $\mathrm{T}$, Shigematsu $\mathrm{T}$, Takahashi S. Kimura's disease and its relation to angiolymphoid hyperplasia with eosinophilia: report of three cases and review of the literature. J Oral Pathol. (1986) 15:132-7. doi: 10.1111/j.1600-0714.1986.tb0 0593.x

5. Park SW, Kim HJ, Sung KJ, Lee JH, Park IS. Kimura disease: CT and MR imaging findings. AJNR Am J Neuroradiol. (2012) 33:784-8. doi: 10.3174/ajnr.A2854

6. Zhou P, Zhang W, Shi W, Zhang Y. Kimura disease. Dermatol Online J. (2017) 23:13030/qt4jx9v026.

7. Gurram P, Chandran S, Parthasarathy P, Thiagarajan MK, Ramakrishnan K. KIMURA'S disease - an e[X]clusive condition. Ann Maxillofac Surg. (2019) 9:183-7. doi: 10.4103/ams.ams_159_17

8. Bastos JT, Rocha CRMD, Silva PMCE, Freitas BMP, Cassia FF, Avelleira JCR. Angiolymphoid hyperplasia with eosinophilia versus Kimura's disease: a case report and a clinical and histopathological comparison. An Bras Dermatol. (2017) 92:392-4. doi: 10.1590/abd1806-4841.201 75318

9. Fite-Trepat L, Martos-Fernandez M, Alberola-Ferranti M, Pablo-GarciaCuenca A, Bescosatin C. Angiolymphoid hyperplasia with eosinophilia involving the occipital artery: case report and review of literature.

\section{ETHICS STATEMENT}

The studies involving human participants were reviewed and approved by Ethics Committee of Shengjing Hospital. The patients/participants provided their written informed consent to participate in this study. Written informed consent was obtained from the individual(s) for the publication of any potentially identifiable images or data included in this article. Informed written consent was obtained from the patient for publication of this report and any accompanying images.

\section{AUTHOR'S NOTE}

The authors have read the CARE Checklist (2016), and the manuscript was prepared and revised according to the CARE Checklist (2016).

\section{AUTHOR CONTRIBUTIONS}

$\mathrm{HZ}, \mathrm{Z}-\mathrm{wG}$, and $\mathrm{Z}-\mathrm{wC}$ reviewed the literature and contributed to manuscript drafting. Z-wG was responsible for revision of the manuscript for important intellectual content. All authors issued final approval for the version to be submitted.
J Clin Diagn Res. (2017) 11:ZD21-3. doi: 10.7860/JCDR/2017/23323. 9569

10. Don DM, Ishiyama A, Johnstone AK, Fu YS, Abemayor E. Angiolymphoid hyperplasia with eosinophilia and vascular tumors of the head and neck. Am J Otolaryngol. (1996) 17:240-5. doi: 10.1016/S0196-0709(96)9 0088-5

11. Rosai J, Gold J, Landy R. The histiocytoid hemangiomas. A unifying concept embracing several previously described entities of skin, soft tissue, large vessels, bone, and heart. Hum Pathol. (1979) 10:707-30. doi: 10.1016/S0046-8177(79)80114-8

12. Su S, Chen X, Li J, Yu J, Zhang L. Kimura's disease with membranoproliferative glomerulonephritis: a case report with literature review. Ren Fail. (2019) 41:126-30. doi: 10.1080/0886022X.2019.15 84115

13. Ren S, Li XY, Wang F, Zhang P, Zhang Y, Li GS, et al. Nephrotic syndrome associated with Kimura's disease: a case report and literature review. $B M C$ Nephrol. (2018) 19:316. doi: 10.1186/s12882-018-1123-y

14. Wang Z, Zhang J, Ren Y, Dai Z, Ma H, Cui D, et al. Successful treatment of recurrent Kimura's disease with radiotherapy: a case report. Int J Clin Exp Pathol. (2014) 7:4519-22.

15. Lee J, Hong YS. Kimura disease complicated with bowel infarction and multiple arterial thromboses in the extremities. $J$ Clin Rheumatol. (2014) 20:38-41. doi: 10.1097/RHU.00000000000 00064

16. Ye P, Ma DQ, Yu GY, Gao Y, Peng X. Comparison of the efficacy of different treatment modalities for Kimura's disease. Int J Oral Maxillofac Surg. (2017) 46:350-4. doi: 10.1016/j.ijom.2016.08.013

17. Zhang G, Li X, Sun G, Cao Y, Gao N, Qi W. Clinical analysis of Kimura's disease in 24 cases from China. BMC Surg. (2020) 20:1. doi: 10.1186/s12893-019-0673-7

18. Chang AR, Kim K, Kim HJ, Kim IH, Park CI, Jun YK. Outcomes of Kimura’s disease after radiotherapy or nonradiotherapeutic treatment modalities. Int J Radiat Oncol Biol Phys. (2006) 65:1233-9. doi: 10.1016/j.ijrobp.2006. 02.024 
19. Chen QL, Dwa S, Gong ZC, Abasi K, Ling B, Liu H, et al. Kimura's disease: risk factors of recurrence and prognosis. Int J Clin Exp Med. (2015) 8:21414-20.

20. Eh Dam VSK, Mohamad S, Mohamad I. Kimura disease with parotid swelling and cervical lymphadenopathy: a case report and literature review. Medeni Med J. (2020) 35:170-4. doi: 10.5222/MMJ.2020. 84594

21. Dai L, Wei XN, Zheng DH, Mo YQ, Pessler F, Zhang BY. Effective treatment of Kimura's disease with leflunomide in combination with glucocorticoids. Clin Rheumatol. (2011) 30:859-65. doi: 10.1007/s10067-0111689-2
Conflict of Interest: The authors declare that the research was conducted in the absence of any commercial or financial relationships that could be construed as a potential conflict of interest.

Copyright (๑) 2021 Zhao, Cao and Gu. This is an open-access article distributed under the terms of the Creative Commons Attribution License (CC BY). The use, distribution or reproduction in other forums is permitted, provided the original author(s) and the copyright owner(s) are credited and that the original publication in this journal is cited, in accordance with accepted academic practice. No use, distribution or reproduction is permitted which does not comply with these terms. 